\title{
A 3-D mid-infrared view of the central parsec
}

\author{
J. Moultaka ${ }^{1}$, A. Eckart ${ }^{2},{ }^{3}$, K. Mužić ${ }^{4}$ and N. Sabha ${ }^{2}$ \\ ${ }^{1}$ Université de Toulouse III - CNRS - IRAP, Observatoire Midi-Pyrénées \\ 14, Avenue Edouard Belin 31400 Toulouse, France \\ email: jihane.moultaka@irap.omp.eu \\ ${ }^{2}$ I. Physikalisches Institut, Universität zu Köln, Zülpicher Str. 77, 50937 Köln, Germany \\ ${ }^{3}$ Max Planck Institut für Radioastronomie, Auf dem Hügel 69, 53121 Bonn, Germany \\ ${ }^{4}$ ESO, Alonso de Cordova 3107, Vitacura, Casilla 19, Santiago, 19001, Chile
}

\begin{abstract}
We present a mid-infrared 3D view of the central parsec using ISAAC spectrograph (ESO/VLT) with its spectroscopic mode. We mapped the central parsec in $L$ - and $M$-bands by using 27 and 21 slit positions, respectively, that allowed us to build two data cubes of the region in these spectral domains. We also use an original method to distinguish the contribution of the foreground extinction to the absorbed spectra from that of the local extinction in both wavelength ranges. We find that there are residual water and $\mathrm{CO}$ ices in the central parsec as well as hydrocarbons and gaseous CO implying very low temperatures of the order of tens of Kelvin in the local environment of Sgr A*.
\end{abstract}

Keywords. Galaxy: center — infrared: ISM — ISM: molecules

\section{Observations}

We used the ISAAC spectrograph located at the ESO UT3-VLT telescope in order to map the central parsec of our Galaxy in the $L$ - and $M$-band wavelength ranges (i.e. from $2.8 \mu \mathrm{m}$ to $4.2 \mu \mathrm{m}$ and $4.6 \mu \mathrm{m}$ to $4.76 \mu \mathrm{m}$ respectively). We needed 27 and 21 slit positions, respectively, to map the desired region with a seeing varying from $0.4^{\prime \prime}$ to $1.5^{\prime \prime}$. This resulted in the first data cubes of the region in these wavelength ranges.

\section{Correcting the line of sight extinction}

In Moultaka et al. (2004), we derive a spectrum of the foreground extinction in the wavelength range of the SL filter of ISAAC (see the paper for more details). In Moultaka et al. (2009), we derive a spectrum of the foreground contribution from the solid-phase ${ }^{12} \mathrm{CO}$ absorption line located at $4.675 \mu \mathrm{m}$ (see figure and explanation in that paper). We show that this spectrum diluted with an additional continuum of about $4 \mathrm{Jy}$ is well suited to describe the foreground overall extinction in the spectral region. We corrected the data cubes for the foreground extinction by dividing them with the corresponding spectrum. In the case of the $M$-band data cube, we added a $4 \mathrm{Jy}$ continuum to the calibrator spectrum.

\section{Results and conclusion}

From the corrected $L$-band data cube for the foreground extinction, we built the optical depth map of the water ice absorption feature at $3.0 \mu \mathrm{m}$ and that of the hydrocarbon absorption at $3.4 \mu \mathrm{m}$ (we show in Figure 1a the water ice map). On the other hand, we 

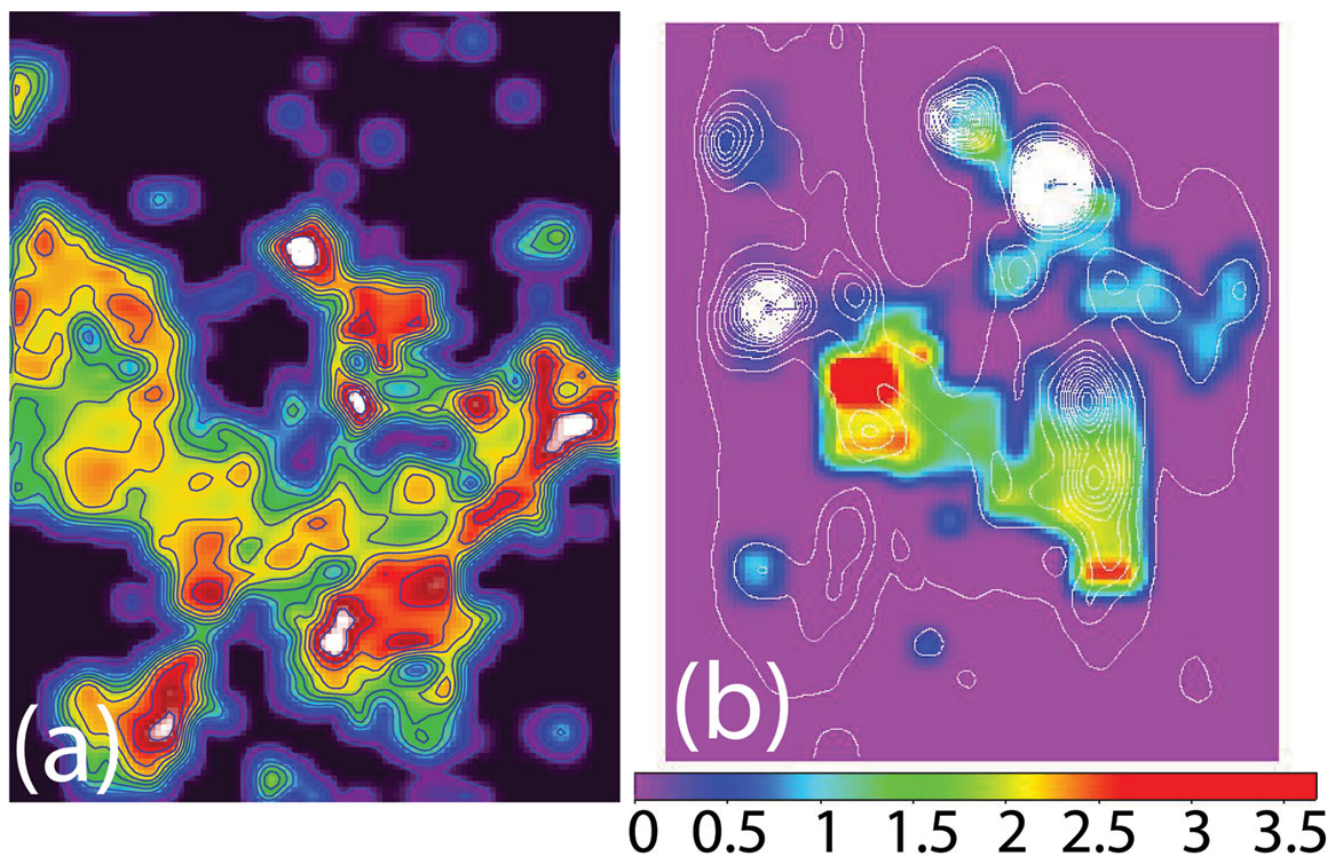

Figure 1. (a) Map of the optical depths of the water ice absorption feature at $3.0 \mu \mathrm{m}$. Contours are also shown for clarity. (b) Map of the optical depths ratio of the solid ${ }^{12} \mathrm{CO}$ line to gaseous ${ }^{12} \mathrm{CO} \mathrm{P}(2)$ line corrected for the foreground ${ }^{12} \mathrm{CO}$ line contribution. Contours of the continuum $M$-band integrated flux are overlaid. [A COLOR Version is AVAilable online.]

constructed the optical depth map of the gaseous ${ }^{13} \mathrm{CO}-\mathrm{R}(0)$ line (located at $4.765 \mu \mathrm{m}$ ) using the $M$-band data cube corrected for the foreground solid ${ }^{12} \mathrm{CO}$ extinction. The resulting maps agree well with our previous work presented in Moultaka et al. (ApJ 703, 2009) on individual sources of the Galactic center. For IRS16C, we find a value of about 0.1 in the corrected map which was the value we used in that paper to correct all the spectra for the gaseous foreground absorption.

We derive the optical depth map of the solid-phase ${ }^{12} \mathrm{CO}$ line and find residuals with optical depth values ranging from 0.1 to 0.9 . These residuals can be due to the gaseous ${ }^{12} \mathrm{CO} \mathrm{P}(1)$ line which is located at the same wavelength (see figure 8 in Moultaka et al. 2009 taken from Moneti et al. 2001 and plotted at our spectral resolution). In this figure, we measure the ratio between the optical depths of the ${ }^{12} \mathrm{CO} \mathrm{P}(1)$ and the ${ }^{12} \mathrm{CO} \mathrm{P}(2)$ lines and find 1.05. We can thus compare the same ratio in our maps with this value and conclude that if this ratio is higher than 1.05, then there is a residual solid-phase ${ }^{12} \mathrm{CO}$ line. In Figure 1b, we show the optical depth ratio map of the solid ${ }^{12} \mathrm{CO}$ to gaseous ${ }^{12} \mathrm{CO} \mathrm{P}(2)$ line corrected for the foreground ${ }^{12} \mathrm{CO}$ line contribution. This figure shows clearly that the ratio is much higher than 1.05, especially in the southern and western parts of the maps implying residual solid ${ }^{12} \mathrm{CO}$ line.

Our data show large quantities of water ice and solid ${ }^{12} \mathrm{CO}$, hydrocarbons and gaseous $\mathrm{CO}$ in the local environment of the central parsec. This implies very low temperatures of less than $60 \mathrm{~K}$ in the region surrounding the supermassive black hole Sgr A*.

The presence of bow shock sources (e.g., Tanner et al. 2002, Tanner et al. 2005), narrow dust filaments (Muzic et al. (2007)), and dust-embedded YSOs (IRS 13N; Muzic et al. 2008) in the central parsec, suggest high-density pockets and high-optical depths. At such 
high optical depths, gas temperatures can fall below the dust temperatures of $200 \mathrm{~K}$ on average in the minispiral and can explain the presence of these ices.

\section{References}

Moneti, A., Cernicharo, J., \& Pardo, J. R. 2001, ApJ 549, 203

Moultaka, J., Eckart, A., Viehmann, Th., Mouawad, N., et al. 2004, A $\xi A$ 425, 529

Moultaka, J., Eckart, A., \& Schödel, R. 2009, ApJ 703, 1635

Muzic, K., Eckart, A., Schödel, R., Meyer, L., \& Zensus, A. 2007, A $\& A$ 469, 993

Muzic, K., Schödel, R., Eckart, A., Meyer, L., \& Zensus, A. 2008, A\& $A$ 482, 173

Tanner, A., Ghez, A. M., Morris, M., Becklin, E. E., Cotera, A., et al. 2002, ApJ 575, 860

Tanner, A., Ghez, A. M., Morris, M. R., \& Christou, J. C. 2005, ApJ 624, 742 